\title{
Superconductivity Modulated by Binary Doping in $\mathrm{Nd}_{1-\mathrm{x}} \mathrm{Ba}_{\mathrm{x}} \mathrm{FeAsO}_{1-2 \mathrm{x}} \mathrm{F}_{2 \mathrm{x}}$
}

\author{
Chunqing $Q u^{1,2}$, Zhiyong Liu ${ }^{1}$, Yuming Lu ${ }^{1}$, Changzhao Chen ${ }^{1}$, Chuanbing Cai ${ }^{1}$, Aihua Fang ${ }^{3}$, \\ Fuqiang Huang ${ }^{3}$, Mingfeng $\mathrm{Wang}^{2}$, Xiaoming Xie ${ }^{2}$ \\ ${ }^{1}$ Research Center for Superconductors and Applied Technologies, Physics Department, \\ Shanghai University, Shanghai, China \\ ${ }^{2}$ State Key Laboratory of Functional Materials for Informatics, Shanghai Institute of Microsystem and \\ Information Technology, Chinese Academy of Sciences, Shanghai, China \\ ${ }^{3}$ Shanghai Institute of Ceramics, Chinese Academy of Sciences, Shanghai, China \\ E-mail: cbcai@staff.shu.edu.cn \\ Received February 12, 2011; revised March 17, 2011; accepted April 12, 2011
}

\begin{abstract}
Binary doping effect is studied for the Fe-based superconductors of $\mathrm{Nd}_{1-\mathrm{x}} \mathrm{Ba}_{\mathrm{x}} \mathrm{FeAsO}_{1-2 \mathrm{x}} \mathrm{F}_{2 \mathrm{x}}(\mathrm{x}=0.02,0.05,0.1$, $0.15,0.2)$. The $\mathrm{X}$-ray diffractions show that the $c$-axis lattice constant decreases monotonously with the doping content, in contrast to the little change in the $a$-axis. Temperature dependences of electric resistivity and magnetic susceptibility reveal that the superconductivity for the studied system emerges at $\mathrm{x}=0.1$, and enhances together with $H_{c 2}(0)$ as the doping content $\mathrm{x}$ increases further. In case of $\mathrm{x}=0.2$, the superconducting critical temperature reaches as high as $50 \mathrm{~K}$, which is the first demonstration of superconductivity with a high fluorine-doping induced by both electron and hole doping in this family. Negative Hall coefficient $\left(R_{H}\right)$ indicates that electron-type carriers are dominated in the present samples. The complicated temperature dependence of $R_{H}$, is believed to arise from a multiband effect together with a complicated scattering, especially at the temperature near the $T_{c}$.
\end{abstract}

Keywords: Binary Doping, Iron-Based Superconductor, Flux Pinning, Scattering

\section{Introduction}

The fantastic discovery of superconductivity with the transition temperature $T_{\mathrm{c}}$ of around $26 \mathrm{~K}[1]$ in the new class of iron-arsenide has attracted wide interest in the world. Among the family of Fe-based superconductors, the $\mathrm{LaFeAsO}_{1-\mathrm{y}}(\mathrm{FeAs}-1111)$ exhibits quite high critical temperatures, such as $T_{\mathrm{c}}=55 \mathrm{~K}$ [2] in the case of electron doping like $\mathrm{SmFeAsO}_{1-\mathrm{x}} \mathrm{F}_{\mathrm{x}}$, and $T_{c}=25 \mathrm{~K}$ [3] in the case of hole doping like $\mathrm{La}_{1-\mathrm{x}} \mathrm{Sr}_{\mathrm{x}} \mathrm{FeAsO}$.

It is well known that there are other types of ironpnictide superconductors, among which the $\mathrm{FeAs}_{1-\mathrm{y}}$ (FeAs-11), a type of Fe-chalcogenide superconductor, attracts a lot of attentions due to its simple structure and composition [4]. It is reported that the FeAs- 11 exhibits the zero resistance transition temperature of $8 \mathrm{~K}$ for the PbO-type $\alpha$-FeSe compound [5], while the $\mathrm{Li}_{x} \mathrm{FeAs}$ (FeAs-111), as an infinite layered structure [6,7] may exhibit the $T_{c}$ of $18 \mathrm{~K}$ without SDW phases.

In contrast, the FeAs-1111 involves two types of par- ent compounds [1], i.e., $\mathrm{LnFeAsO}$ ( $\mathrm{Ln}$ is rare earths) and $\mathrm{AFeAsF}$ (A is positive bivalent ion), both exhibiting the tetragonal space group $P 4 / \mathrm{nmm}$ at $300 \mathrm{~K}$. The first one shows an anomaly of magnetic phase transition near 150 $\mathrm{K}$, which is believed to be caused by the spin-densitywave (SDW) instability [8]. This kind of Fe-based superconductor is characterized as electron-doped [9-13] and its $T_{c}$ may be enhanced to be $55 \sim 56 \mathrm{~K}$ via replacing lanthanum with other rare-earth elements. [2,9-14] The second parent compound such as SrFeAsF being of ZrCuSiAs-tpye structures [15,16], shows an SDW and/or structural transition at about $173 \mathrm{~K}$, and its positive Hall coefficient $\left(R_{\mathrm{H}}\right)$ suggesting that the conduction of this parent phase is dominated by hole-like charge carriers. [14]

According to existing investigations, the parent compounds of both $\mathrm{LnFeAsO}$ and $\mathrm{AFeAsF}$ show the SDW but no superconductivity, and they can evolve into superconductors as a consequence of the normative symmetry breaking due to the element doping, such as 
$\mathrm{Gd}_{1-\mathrm{x}} \mathrm{Th}_{\mathrm{x}} \mathrm{FeAsO}$ [13] $\mathrm{SmFe}_{0.9} \mathrm{Co}_{0.1} \mathrm{AsO}$ [17], CeFeAs ${ }_{1-\mathrm{x}}$ $\mathrm{P}_{\mathrm{x}} \mathrm{O}$ [18], and $\mathrm{LaFeAsO}_{1-\mathrm{x}} \mathrm{F}_{\mathrm{x}}$ [1]. Similar phenomena also exists in FeAs-122 system, such as $\mathrm{Ca}_{1-\mathrm{x}} \mathrm{Na}_{\mathrm{x}} \mathrm{Fe}_{2} \mathrm{As}_{2}$ [19], $\mathrm{Ba}\left(\mathrm{Fe}_{0.961} \mathrm{Ru}_{0.039}\right)_{2} \mathrm{As}_{2}$ [20], $\mathrm{EuFe}_{2}\left(\mathrm{As}_{0.7} \mathrm{P}_{0.3}\right)_{2}$ [21] and so on.

With respect to the FeAs-1111 system, the fluorine doping or oxygen deficiency related to the oxygen-site is frequently studied $[22,23]$, disclosing that they are effective to produce the superconductivity. For FeAs-11 system, it is reported that the clean superconducting phase exists only in those samples with intentional Se deficiency [24,25], and single crystal samples are hardly obtained with a stoichiometric composition of 1:1. All of these suggest that the superconductivity may emerge or be enhanced by element substitution due to normative symmetry breaking in electronic structure, charge or stress etc.

Apart from one element or one site substitution, it is also reported that the second element doping at other site is applicable to modify the normative symmetry and then the superconductivity [26-28], such as $\mathrm{Ce}_{1-\mathrm{x}} \mathrm{Gd}_{\mathrm{x}}$ $\mathrm{FeAsO}_{0.84} \mathrm{~F}_{0.16}$ [26], and $\mathrm{SmFe}_{1-\mathrm{x}} \mathrm{Ru}_{\mathrm{x}} \mathrm{AsO}_{0.85} \mathrm{~F}_{0.15}$ [27].

Compared with the case of one site doping, two element substitutions or doping may exhibit more unique phenomenon. In the present paper, we focus on the FeAs-1111 system and study its doping effect using binary substitution, namely $\mathrm{Nd}_{1-x} \mathrm{Ba}_{x} \mathrm{FeAsO}_{1-2 \mathrm{x}} \mathrm{F}_{2 x}$. This special system with both electron-doping and hole-doping allow more breaking of original symmetry, and this may give rise to complicate modification for superconducting and magnetic behaviors. In the flowing sections, the structural and electromagnetic characteristics regarding this system are systemically investigated, to understand its distinct performances and underlying mechanism due to binary doping.

\section{Experimental}

Polycrystalline bulks with nominal compositions of $\mathrm{Nd}_{1-\mathrm{x}} \mathrm{Ba}_{\mathrm{x}} \mathrm{FeAsO}_{1-2 \mathrm{x}} \mathrm{F}_{2 \mathrm{x}}(\mathrm{x}=0.02,0.05,0.1,0.15$, and 0.2) were synthesized by conventional solid-state reaction. Firstly, the intermediate phases of $\mathrm{Fe}_{2} \mathrm{As}, \mathrm{NdAs}$ and $\mathrm{FeO}$ were prepared by the reactions of the mixed commercial powders of $\mathrm{Nd}(99.5 \%), \mathrm{Fe}(99.9 \%)$, As $(99.999 \%)$ and $\mathrm{Fe}_{2} \mathrm{O}_{3}(99.9 \%)$, sealed in a vacuum quartz tube, annealed at $600^{\circ} \mathrm{C}$ for $6 \mathrm{~h}$ and then $850^{\circ} \mathrm{C}$ for $12 \mathrm{~h}$.

Subsequently, the resultant precursors together with $\mathrm{BaF}_{2}$ powder in stoichiometry of $\mathrm{Nd}_{1-\mathrm{x}} \mathrm{Ba}_{\mathrm{x}} \mathrm{FeAsO}_{1-2 \mathrm{x}} \mathrm{F}_{2 \mathrm{x}}$ were ground for $15 \mathrm{~min}$. All the weighing and mixing procedures were performed in a glove box filled with high purity Ar gas. After that, the mixtures pressed to into pellets in diameter of $8 \mathrm{~mm}$, were sealed in a vacuum quartz tube, and then heated up to $1160^{\circ} \mathrm{C}$ by a rate of $230^{\circ} \mathrm{C} / \mathrm{h}$. For the reference, the pellets are divided into two sorts, with and without surface wrapping by $\mathrm{Ta}$ pieces [29] to test the effect of preventing fluorine-volatilizing during the solid-state reaction.

Note that the present precursors with and without Ta wrapping were maintained at the temperature of $1160^{\circ} \mathrm{C}$ for $10 \mathrm{~h}$ only, rather than several ten hours reported by others. This is purposely selected as we believe that it is critical to shorten the heating time to avoid the fluorine-volatilizing at high temperatures, and to increase the synthesis efficiency as well.

The crystal structure and phase purity of all studied samples were characterized by X-ray diffraction (XRD). The superconducting properties were measured by using a physical property measurement system (Quantum Design PPMS) in magnetic fields up to $9 \mathrm{~T}$. Magnetic and transport performances are evaluated by a series of characteristic curves including the temperature dependences of resistivity, dc susceptibility, and the Hall coefficient etc. For the magnetization measurements, rectangular specimens were prepared nearly in the same dimension of about $6 \times 2 \times 1 \mathrm{~mm}^{3}$, and the applied magnetic fields were normal to the long axis direction of the specimen.

\section{Result and Discussion}

\subsection{Microstructure, Lattice Constants and Their Doping Dependences}

The XRD patterns for the samples with various doping contents of $0.02-0.2$ are shown in Figure 1. It is clear that the main peaks for all studied samples can be indexed to the FeAs-1111 phase with the tetragonal ZrCuSiAs-type structure, regardless of the slight shift of main peaks and several weak peaks corresponding with the impurity phase of NdOF.

The doping dependence of the lattice constants calculated from the XRD data are presented in Figure 2. There is little change in the lattice constants of $a$-axis as the doping content increases. This may result from the contrary contributions to the in-plane lattice from two elements doping, since the ionic radius of $\mathrm{Ba}^{2+}$ is larger than that of $\mathrm{Nd}^{3+}$, while the ionic radius of $\mathrm{F}^{-}$is smaller than that of $\mathrm{O}^{2-}$. In contrast, the $c$-axis lattice constants decrease monotonously with the doping content. Such a reduction probably arises from the decrease in layer distance between FeAs and $\mathrm{NdO}$ which may take places due to chemical pressure variation along the $c$-axis [30]. Note that this is unlike the case of hole-like system such as $\mathrm{Pr}_{1-\mathrm{x}} \mathrm{Sr}_{\mathrm{x}} \mathrm{FeAsO}$ [31] and $\mathrm{Nd}_{1-\mathrm{x}} \mathrm{Sr}_{\mathrm{x}} \mathrm{FeAsO}$ [32], where the $c$-axis lattices increase monotonously with increasing the doping content of $\mathrm{Sr}$. It is thus observed that the $c$-axis lattice increase with the doping content is mostly present 


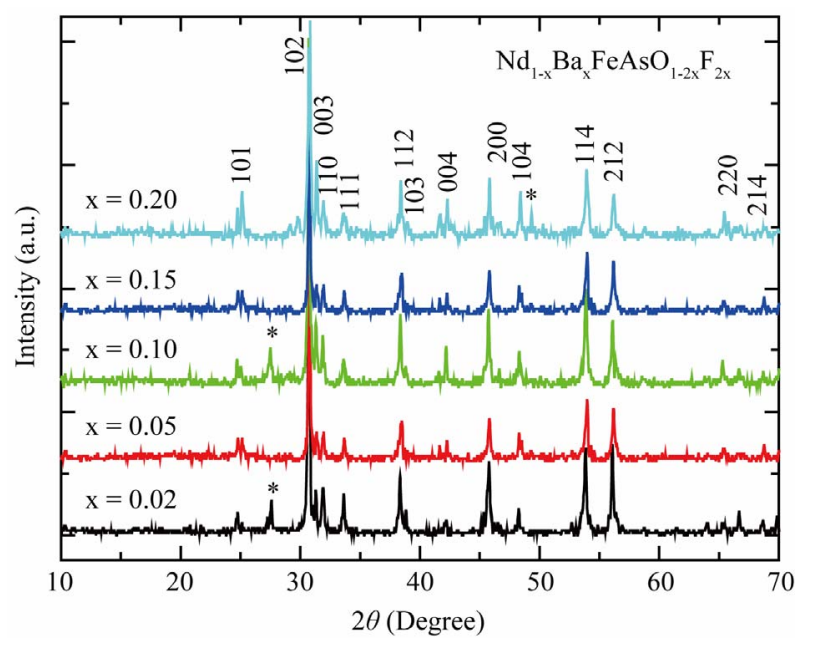

Figure 1. (Colour online) $\mathrm{X}$-ray diffraction patterns for the $\mathrm{Nd}_{1-\mathrm{x}} \mathrm{Ba}_{\mathrm{x}} \mathrm{FeAsO}_{1-2 \mathrm{x}} \mathrm{F}_{2 \mathrm{x}}$ compounds with various doping contents from $x=0.02$ (bottom) to $x=0.2$ (top).

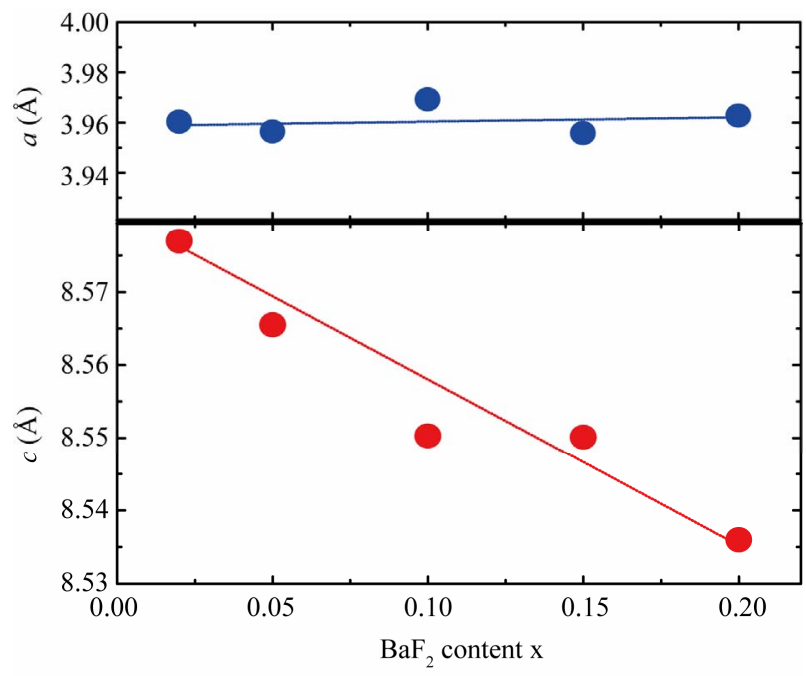

Figure 2. (Colour online) Doping content dependence of $a$-axis and $c$-axis lattice constants.

in hole-like doping FeAs-1111 system, while $c$-axis decrease with the doping mostly in electron-like doping. With respect to the chemical pressure change, it is supposed that a further increase in $T_{c}$ may emerge with more $\mathrm{BaF}_{2}$ chemically doped into the FeAs-1111 system [33].

\subsection{Phase Diagram Regarding Superconductivity and SDW Transition}

Characteristic transition temperatures are evaluated by both $R-T$ curve and dc susceptibility measurements. Figure 3(a) shows the $R-T$ curves for the samples with various doping contents prepared by solid-state reaction without Ta wrapping. To test the stability of the samples, the $R-T$ curves for all samples are measured twice, i.e., immediately after growing and after five months in air, which show a similar transport behavior, suggesting no obvious performance degradation with time going. The inset of Figure 3(a), shows two $R-T$ curves for the same sample of $\mathrm{x}=0.15$, measured before and after five months to give a direct comparison.

Figure 3(b) shows the superconducting critical temperature as well as its transition width, identified from the $R$ - $T$ curves in Figure 3(a). It is revealed that the present samples exhibit the superconductivity as $\mathrm{x}$ is more than 0.1 , being as high as $50 \mathrm{~K}$ at $\mathrm{x}=0.2$. Moreover, the superconducting transition width $\Delta T$, determined by the temperatures corresponding to $90 \%$ and $10 \%$ of the normal-state resistivity, decreases with increasing doping content, being as low as $1.86 \mathrm{~K}$ at $\mathrm{x}=0.2$.

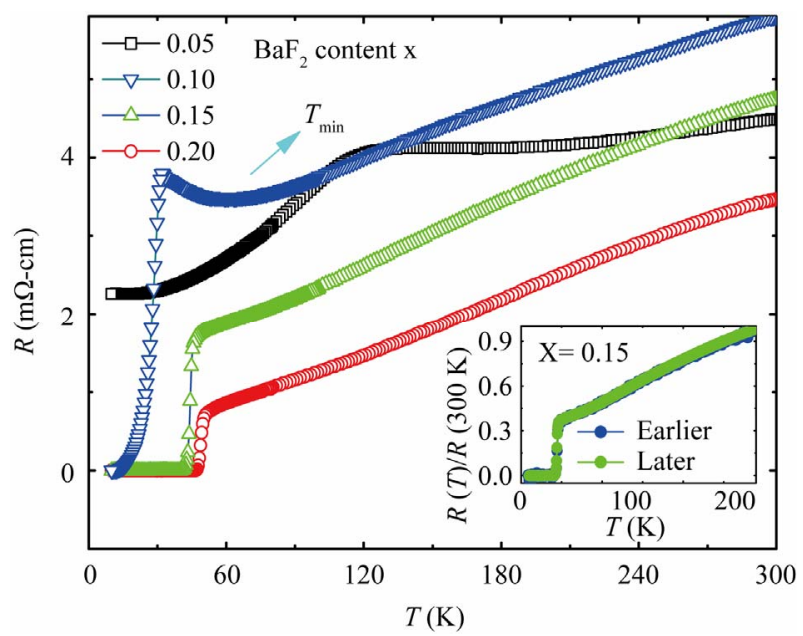

(a)

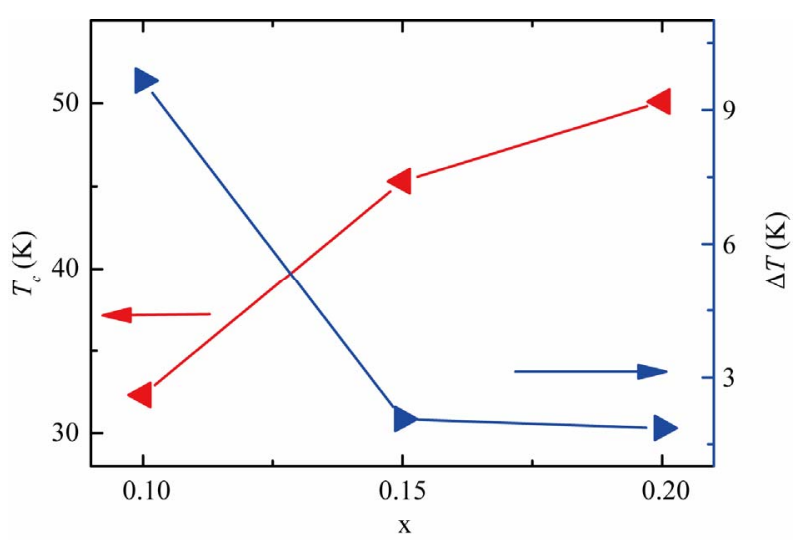

(b)

Figure 3. (colour online) (a) Temperature dependence of resistivity for the studied samples with $x=0.05,0.1,0.15$, and 0.2 . Inset shows two $R-T$ curves for the same sample of $\mathbf{x}=0.15$, measured before and after 5 months, respectively; (b) The doping content dependence of $T^{\text {onset }}$ and $\Delta T$ (determined by the difference of $T_{c}^{90 \%}$ and $T_{c}^{10 \%}$. 
To further confirm the transport results, the temperature dependences of dc susceptibility are measured by ZFC (zero field cooling) and FC (field cooling) modes with an excitation field of 100oe. Figure 4 shows the temperature dependences of the volume magnetic susceptibility for two typical samples of $\mathrm{x}=0.15$ and $\mathrm{x}=$ 0.2 , demonstrating their onset superconducting critical temperatures of $44.2 \mathrm{~K}$ and $48.8 \mathrm{~K}$, respectively.

Note that the $T_{c}$ measured by dc susceptibility is a little lower than the values measured by transport due to percolation effect. They are, however, close to the values of $T_{c}^{50 \%}$ dominated by the $50 \%$ of the normal state resistivity, which is frequently observed for Fe-base superconductors. Moreover, the width of magnetic transition is generally larger than that of resistivity, which is due to the magnetic signal out of statistic results for whole grains, being more sensitive than the transport.

Apart from superconducting transition temperatures, another characteristic temperature $T_{S}$ regarding the phase transition of SDW, can be identified as well based on the temperature dependences of resistance and dc susceptibility. For the sample of $x=0.05$, no superconductivity is observed, while a SDW transition temperature of $117 \mathrm{~K}$ is clearly demonstrated.

Figure 5 shows a phase diagram with respect to the doping content dependence of characteristic temperatures, including the SDW transition temperature $T_{s}$ and the superconducting transition temperature $T_{c}$ for all studied samples. It is revealed that the samples with and without Ta wrapping exhibit similar dependence of the doping content. The superconductivity appears at $\mathrm{x}>0.1$, and it still exists at a high doping content, e.g. $x=0.2$. While the $T_{\mathrm{s}}$ is hardly affected by wrapping condition, the $T_{c}$ for the sample without wrapping is generally high than the sample with wrapping. This implies that a different wrapping way will lead to a different $T_{c}$, regardless of identical composition and heating route, This is probably due to suppressed fluorine-evaporating as well as F doping content in the case of wrapping, which reminds us of selecting the samples only without Ta wrapping, which give a relatively high $T_{c}$, for more meaningful discussions below.

For the samples of $\mathrm{x}=0.15$ and $\mathrm{x}=0.2$, a clear superconducting transition is observed due to effective fluorine doping, and a metallic characteristic in normal state with a good linear dependence of $R-T$. The sample of $\mathrm{x}=$ 0.1 , however, shows a little complicated temperature dependence of resistance, as this compound lies the boundary of the superconducting area in the phase diagram. An upturn peak of resistance occurs at the temperatures close to $T_{c}$, followed by a semiconductor-like behavior, and then a metallic characteristic as the temperature increases.

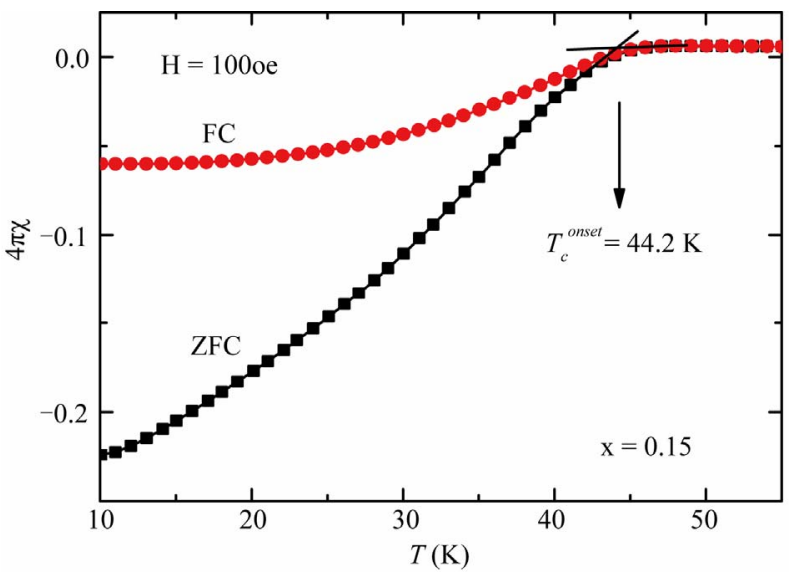

(a)

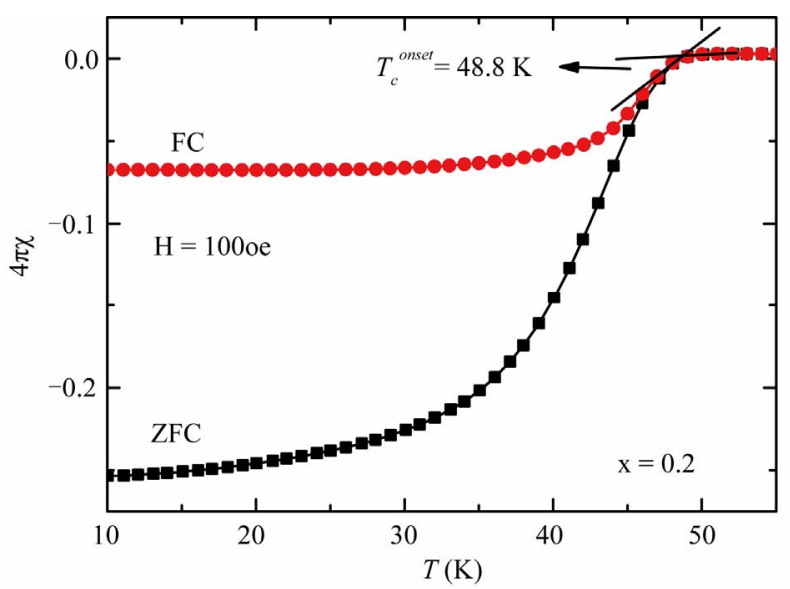

(b)

Figure 4. (Colour online) Temperature dependences of ZFC and FC magnetization curves for the samples of $x=0.15$ (a) and $\mathrm{x}=0.2$ (b).

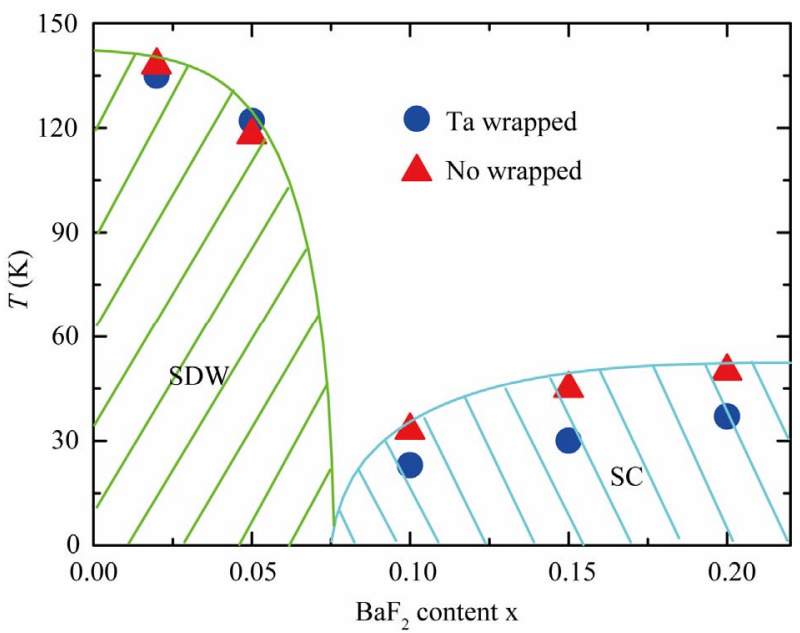

Figure 5. (Colour online) Phase diagram with respect to the superconducting and SDW Transition, determined by the doping dependence of characteristic temperatures for the samples with or without Ta wrapping treatment. 
Similar behavior was also observed in $\mathrm{NdFe}_{1-\mathrm{x}} \mathrm{Rh}_{\mathrm{x}} \mathrm{AsO}$ [34] and it was assumed that such a semiconductor-like behavior actually was one of natures of this family, which was unambiguously proved by band structure calculations as well as photoemission spectroscopy experiments [35]. For the present case, as $x$ increases, both the $T_{c}$ and $\Delta T$ are improved due to the increase of $\mathrm{BaF}_{2}$ doping content, and then a more rigid metallic characteristic that eventually hides the semiconductor-like nature at all. This assumption is in consistent with the observation of the residual resistance ratio $\left(R R R=R(300 \mathrm{~K}) / R\left(T_{c}\right)\right.$, which is enhanced from 1.93 to 5.5 as the $\mathrm{BaF}_{2}$ doping content of $\mathrm{x}$ increases from 0.1 to 0.2 .

\subsection{Characteristic Magnetic Fields}

Figure 6 shows the temperature dependences of resistivity under different magnetic fields for the sample of $\mathrm{x}=$ 0.15 and 0.2 . Based on the magnetotransport measurements, two types of characteristic magnetic fields, irreversibility fields $\left(H_{i r r}\right)$ and upper critical fields $\left(H_{c 2}\right)$ are

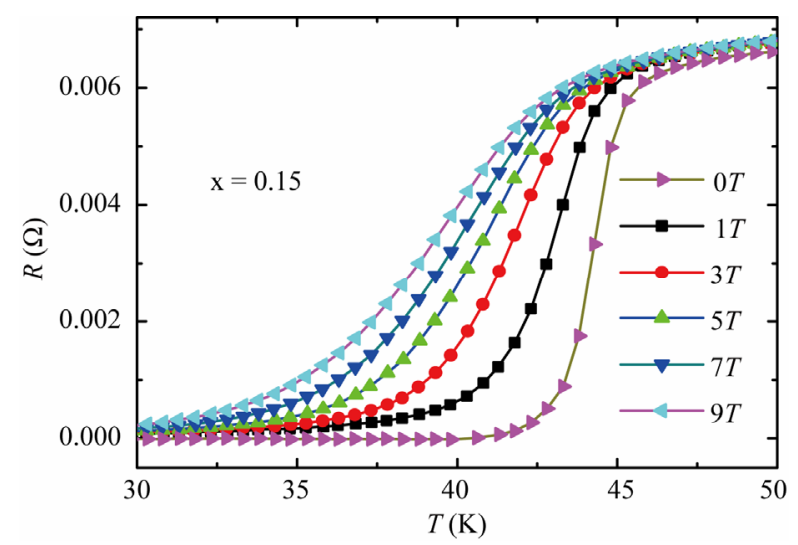

(a)

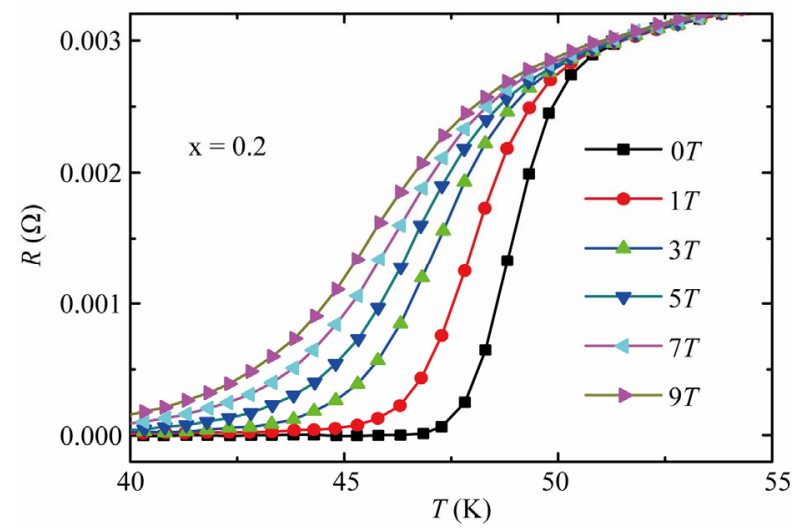

(b)

Figure 6. (Colour online) Temperature dependences of resistance under various magnetic fields for two samples of $\mathrm{x}=0.15$ (a) and $\mathrm{x}=0.2$ (b). determined by the criterion of $10 \%$ and $90 \%$ of normal-state resistivity, respectively. Note that similar evaluation are widely applied to $\mathrm{YBaCuO}, \mathrm{MgB}_{2}$, the F-doped $\mathrm{LaFeAsO}$ and $\mathrm{Sr}$-doped $\mathrm{PrFeAsO}$ polycrystalline samples $[31,36,37]$.

Figure 7(a) shows the relations between the reduced temperature and characteristic magnetic fields. It is revealed that both $H_{i r r}$ and $H_{c 2}$ are enhanced with increasing $\mathrm{x}$ from 0.1 to 0.2 . In case of $\mathrm{x}=0.2$, the $H_{\text {irr }}(T)$ curve is closer to $H_{c 2}(\mathrm{~T})$ curve, which implies that the stronger flux pinning effect hinders the dissipating resistance due to flux jumping.

Figure 7(b) shows the slopes of $H_{c 2}(T)$ near $T_{c}$ for several superconducting samples with higher doping content of x. By using the Werthamer-Helfand Hohenberg (WHH) theory, the zero temperature upper critical field $H_{c 2}(0)$ can be evaluated by the formula below,

$$
H_{c 2}(0)=-0.693 T_{c}\left(\frac{\mathrm{d} H_{c 2}}{\mathrm{~d} T}\right)_{T=T_{c}}
$$

Taking $T_{c}=50 \mathrm{~K}$ for $\mathrm{x}=0.2$ sample, one can get the $H_{c 2}(0)$ of $163 \mathrm{~T}$. As well, $H_{c 2}(0)$ is calculated to be $110 \mathrm{~T}$, and $43 \mathrm{~T}$ for $\mathrm{x}=0.15$ and $\mathrm{x}=0.1$, respectively. Note that these $H_{c 2}(0)$ values are actually lower than that of $\mathrm{SmFeAs}(\mathrm{OF})$ [38], and FeAs-42622 family [16]. However, the slope of $\mathrm{d} H_{c 2} /\left.\mathrm{d} T\right|_{T_{c}}$ for the present sample of $\mathrm{x}=0.2$ is much larger than that of hole doped $\mathrm{Pr}_{1-\mathrm{x}} \mathrm{Sr}_{\mathrm{x}} \mathrm{FeAsO}$ [31], and also than that of electron doped $\mathrm{LaFeASO}_{1-\mathrm{x}} \mathrm{F}_{\mathrm{x}}$ ) [36,37]. Obviously, the values of upper critical field in the case of the binary doping are much more sensitive than that of single element doping. This suggests that the present system with both electron-doping and hole-doping gives rise to more breaking of original symmetry, and then more modification for superconducting behaviors. A direct mechanism may arise from the increased quasiparticle density of states (DOS) near the Fermi level due to both electron and hole doping.

\subsection{Hall Effect and Dominant Carriers}

To get more information about the conducting carriers, the Hall coefficient $\left(R_{H}\right)$ of all samples are measured using the five-probe technique. The deliberated binary doping probably allow more symmetry breaking of the electronic structure, compared with the cases of sole hole doping or electron doping, and this will give rise to the more disorders of internal structure of the compound. Figure 8 shows the temperature dependences of $R_{H}$ for the samples of $\mathrm{x}=0.05,0.10$, and 0.20 . The negative Hall coefficient $R_{H}$ indicates that the electron-type carriers dominate the conduction in the present samples, although the binary doping is purposely designed. This is 


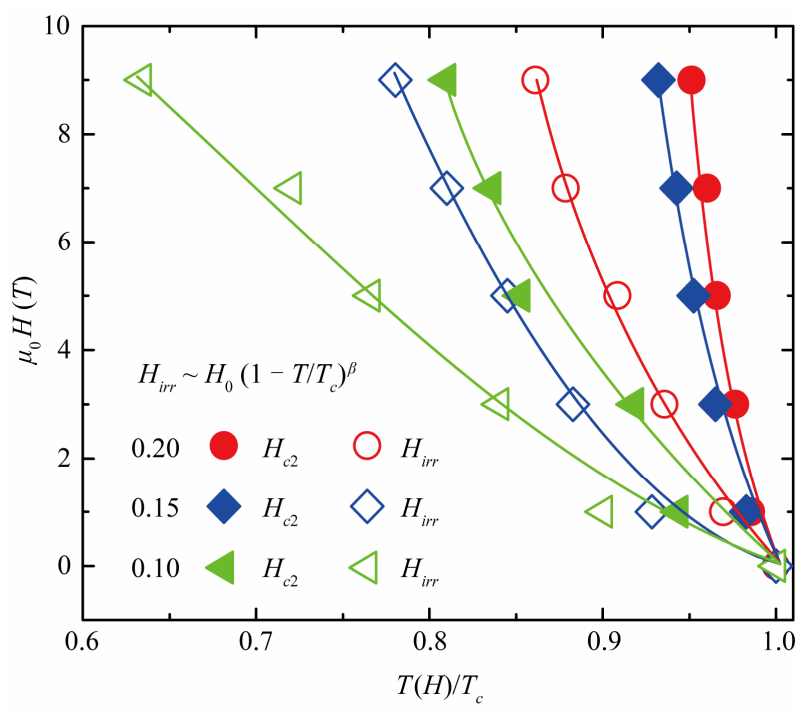

(a)

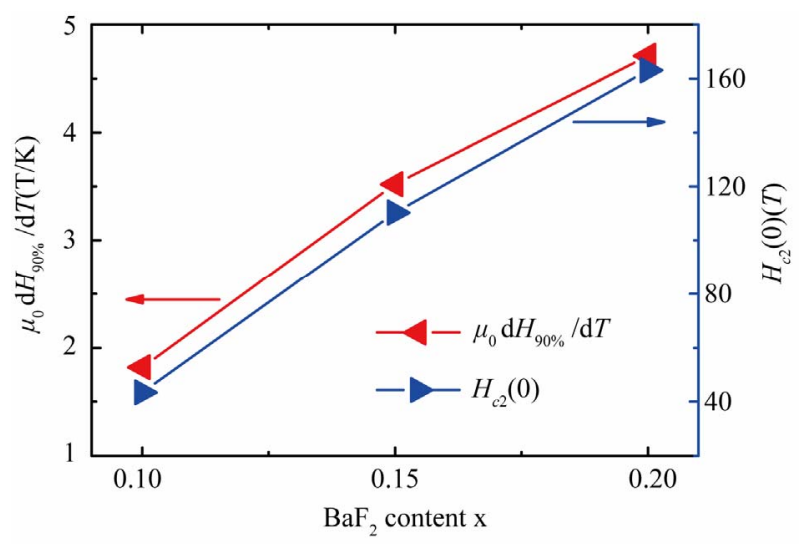

(b)

Figure 7. (Colour online) (a) Reduced temperature dependence of $H_{i r r}$ and $H_{c 2}$ for $x=0.1,0.15,0.2$. (b) Doping content dependence of $\mu_{0} \mathrm{dHd} \mathrm{d}_{90 \%} / \mathrm{dT}$ and $H_{c 2}(0)$; the inset shows the Doping content dependence of $\mu_{0} \mathrm{~d} H \mathrm{~d}_{10 \%} / \mathrm{d} T$.

reasonable if one recall the difference in doping level between Oxygen and Neodymium site. The former is double of the later, leading to the breaking of normative system in electron structure. This is in agreement with the observation of the $c$-axis lattices, which decreases monotonously with increasing the doping content, unlike the case of hole-doped FeAs-1111 system, as discussed in the prior section.

It is well known that the Hall coefficient $R_{H}$ is nearly independent of temperature for conventional metals [31], Whereas, a strong temperature dependence of $R_{H}$ occurs in high-temperature oxide superconductors, and it is regarded as one of the exotic properties. For the present FeAs-based superconductor, the SDW transition may occur at $117 \mathrm{~K}$, while its superconductivity does not emerge at all, such as in the sample of $x=0.05$. For this

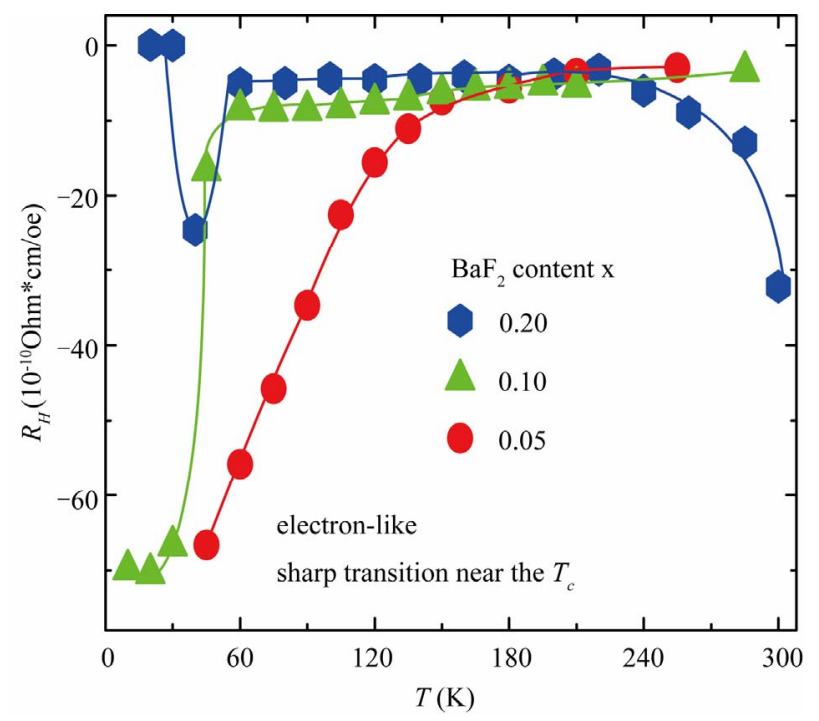

Figure 8. (Colour online) Temperature dependence of Hall coefficiency for the samples of $x=0.05,0.1,0.15,0.2$. It is seen that the $R_{H}$ has a sharp transition near the $T_{c}$, and the electron-type carriers are dominated for all the studied samples.

composition, the temperature dependence of $R_{H}$ is comparatively simple, i.e., the absolute value decreases monotonously with increasing temperature.

A band structure calculation for $\mathrm{LaFePO}$ indicates that all the $d$-orbital energy levels for five $\mathrm{Fe}$ atoms in FeAs-1111 superconductors are not fully occupied, and the cross of the Fermi level $E_{F}$, leads to five Fermi surfaces [39]. Therefore, it is believed that the temperature dependence of $R_{H}$ arises from the multiband effects or magnetic skew scattering mechanism alternatively. Note that the scattering of conduction electrons from local moments is asymmetric due to spin-orbital coupling. A rough estimated from the relation of $n=1 /\left(\mathrm{e}^{*} R_{H}\right)$, indicates the carrier density for our present samples is nearly around $1.6 \times 10^{22} \mathrm{~cm}^{-3}$ at the normal state, being 10 times larger than that of $\mathrm{LaFeAsO}_{0.9} \mathrm{~F}_{0.1-\mathrm{y}}$ [37].

For further increase of $\mathrm{BaF}_{2}$ doping, temperature dependence of $R_{H}$ become stronger, although the monotonous trend still exists. For the present samples of $x=0.1$ and $\mathrm{x}=0.15$, the temperature dependence of $R_{H}$ exhibits a similar behavior. As the temperature increases, the $R_{H}$ jumps from a large negative value to a small constant, which actually corresponds to the phase transition around $T_{c}$.

In the case of $\mathrm{x}=0.2$, the monotonic change disappears and the temperature dependence of $R_{H}$ becomes complicated. Nevertheless, a common feature remains for such a sample, i.e. the $R_{H}$ undergoes a dramatic transition near the superconducting critical temperature. The carrier density slow decrease to $1.2 \times 10^{22} \mathrm{~cm}^{-3}$ at $60 \mathrm{~K}$ from the normal state, when it turns into SC state, the 
value rapid decline to $0.255 \times 10^{22} \mathrm{~cm}^{-3}$ at $40 \mathrm{~K}$, while the further low temperature makes the value dramatically shot up to $6.25 \times 10^{24} \mathrm{~cm}^{-3}$. At the temperature below 30 $\mathrm{K}$, the value of $R_{H}$ is close to zero. As the temperature increases, it becomes a large negative value firstly, and then upturns to be a small constant up to $240 \mathrm{~K}$, and finally goes to increase until $300 \mathrm{~K}$.

The above temperature dependence appears unusual. It, however, is not hard to understand if one recalls the multi-band effect together with a complicated scattering $[3,14,40-42]$, by which a similar scenario in the hole-like $\mathrm{Pr}_{1-\mathrm{x}} \mathrm{Sr}_{\mathrm{x}} \mathrm{FeAsO}$ [31] can be explained well.

It is believed that the present samples follow a strong multi-band effect with a large variation of charge carrier densities as well, especially at the high doping content where the nominal composition of $\mathrm{F}$ reaches as high as $40 \%$. These factors suggest that the resultant $R_{H}$ is probably weighted by the sum of the contributions from each band. Assuming that the scattering rate of each band has different temperature dependence, it is reasonable to see the weighted sum change with temperature. In reality, both the effects are able to make collective contributions to the Hall signal in the present $\mathrm{Nd}_{1-\mathrm{x}} \mathrm{Ba}_{\mathrm{x}}$ $\mathrm{FeAsO}_{1-2 \mathrm{x}} \mathrm{F}_{2 \mathrm{x}}$ since the electrons are dominant carries in this system.

\section{Summary}

Effect of binary doping on the compound of $\mathrm{Nd}_{1-x} \mathrm{Ba}_{\mathrm{x}}$ $\mathrm{FeAsO}_{1-2 \mathrm{x}} \mathrm{F}_{2 \mathrm{x}}$ has been investigated with respect to distinct structural and superconducting properties. It is revealed that the $c$-axis lattice constants decrease monotonously with increasing doping content. In case of $x>0.1$, the magnetic order of SWD may be destroyed and superconductivity takes places, which are confirmed by the measurements of electrical resistivity, and dc susceptibility, and Hall coefficient. The onset superconducting transition temperature can reach $50 \mathrm{~K}$ at $\mathrm{x}=0.2$. The negative Hall coefficient at the studied temperature range suggests that the electron-type charge carriers are dominated, regardless of both electron and hole doping purposely designed. The complicated temperature dependence of $R_{H}$, is believed to be attributed to a multiband effect together with a complicated scattering, especially at low temperature near the $T_{c}$ for such a binary doping system.

\section{Acknowledgements}

This work is partly sponsored by the Ministry of Science and Technology of China (973 Projects, No. 2011CBA 00105 and 863 Projects, No. 2009AA03Z204), the Science and Technology Commission of Shanghai Munici- pality (No. 10dz1203500), and Shanghai Leading Academic Discipline Project (No. S30105). And the supports from the Knowledge Innovation Program of the Chinese Academy of Sciences (Physical Properties and Mechanism of Iron based Superconductors), the Open Project of State Key Laboratory of Functional Materials for Informatics, Chinese Academy of Sciences are gratefully acknowledged as well.

\section{References}

[1] Y. Kamihara, T. Watanabe, M. Hirano and H. Hosono, "Iron-Based Layered Superconductor $\mathrm{La}\left[\mathrm{O}_{1-\mathrm{x}} \mathrm{F}_{\mathrm{x}}\right] \mathrm{FeAs}$ ( $\mathrm{x}=0.05-0.12$ ) with $T_{c} 26 \mathrm{~K}$," Journal of the American Chemical Society, Vol. 130, No. 11, 2008, pp. 32963297. doi: $10.1021 / \mathrm{ja} 800073 \mathrm{~m}$

[2] Z. A. Ren, W. Lu, J. Yang, W. Yi, X. L. Shen, Z. C. Li, G. C. Che, X. L. Dong, L. L. Sun, F. Zhou and Z. X. Zhao, "Superconductivity at $55 \mathrm{~K}$ in Iron-Based F-Doped Layered Quaternary Compound $\mathrm{Sm}\left[\mathrm{O}_{1-\mathrm{x}} \mathrm{F}_{\mathrm{x}}\right] \mathrm{FeAs}$," Chinese Physics Letters, Vol. 25, No. 6, 2008, p. 2215. doi:10.1088/0256-307X/25/6/080

[3] H. H. Wen, G. Mu, L. Fang, H. Yang and X. Y. Zhu, "Superconductivity at $25 \mathrm{~K}$ in Hole-Doped $\left(\mathrm{La}_{1-\mathrm{x}} \mathrm{Sr}_{\mathrm{x}}\right)$ OFeAs," Europhysical Letters, Vol. 82, No. 1, 2008, p. 17009. doi:10.1209/0295-5075/82/17009

[4] Y. Mizuguchi, Y. Takano, "A Review of Fe-Chalcogenide Superconductors: The Simplest Fe-Based Superconductor," Journal of the physical society of Japan, Vol. 79, 2010, p. 102001.

[5] F.-C. Hsu, J.-Y. Luo, K.-W. Ye, T.-K. Chen, T.-W. Huang, P. M. Wu, Y.-C. Lee, Y.-L. Huang, Y.-Y. Chu, D.-C. Yan and M.-K. Wu, "Superconductivity in the PbO-Type Structure-FeSe," Proceedings of the National Academy of Sciences, Vol. 105, No. 38, 2008, pp. $14262-$ 14264. doi:10.1073/pnas.0807325105

[6] X. C. Wang, Q. Q. Liu, Y. X. Lv, W. B. Gao, L. X. Yang, R. C. Yu, F. Y. Li and C. Q. Jin, "The Superconductivity at $18 \mathrm{~K}$ in LiFeAs System," Solid Sate Communications, Vol. 148, No. 11-12, 2008, pp. 538-540. doi:10.1016/j.ssc.2008.09.057

[7] J. H. Tapp, Z. J. Tang, B. Lv, K. Sasmal, B. Lorenz, P. C. W. Chu and A. M. Guloy, "LiFeAs: An Intrinsic FeAsBased Superconductor with T $=18 \mathrm{~K}$," Physical Review B, Vol. 78, No. 6, 2008, p. 4. doi:10.1103/PhysRevB.78.060505

[8] J. Dong, H. J. Zhang, G. Xu, Z. Li, W. Z. Hu, D. Wu, G. F. Chen, X. Dai, J. L. Luo, Z. Feng, N. Fang and N. L. Wang, "Competing Orders and Spin-Density-Wave Instability in $\mathrm{La}\left(\mathrm{O}_{1-\mathrm{x}} \mathrm{F}_{\mathrm{x}}\right) \mathrm{FeAs}$," Europhysics Letters, Vol. 83, No. 2, 2008, p. 27006. doi:10.1209/0295-5075/83/27006

[9] X. H. Chen, T. Wu, G. Wu, R. H. Liu, H. Chen and D. F. Fang, "Superconductivity at $43 \mathrm{~K}$ in $\mathrm{SmFeAsO}_{1-\mathrm{x}} \mathrm{F}_{\mathrm{x}}$," Nature, Vol. 453, No. 7196, 2008, pp. 761-762. doi:10.1038/nature 07045

[10] G. F. Chen, Z. Li, D. Wu, G. Li, W. Z. Hu, J. Dong, P. 
Zheng, J. L. Luo, and N. L. Wang, "Superconductivity at $41 \mathrm{~K}$ and Its Competition with Spin-Density-Wave Instability in Layered $\mathrm{CeO}_{1-\mathrm{x}} \mathrm{F}_{\mathrm{x}} \mathrm{FeAs}$," Physical Review Letters, Vol. 100, No. 24, 2008, Id: 247002.

[11] Z. A. Ren, J. Yang, W. Lu, W. Yi, G.-C. Che, X.-L. Dong, L.-L. Sun and Z.-X. Zhao, "Superconductivity at $52 \mathrm{~K}$ in Iron Based F Doped Layered Quaternary Compound $\mathrm{PO}_{1-\mathrm{x}} \mathrm{F}_{\mathrm{x}} \mathrm{FeAs}$," Materials. Research Innovations, Vol. 12, 2008, p. 105. doi:10.1179/143307508X333686

[12] P. Cheng, L. Fang, H. Yang, X. Y. Zhu, G. Mu, H. Q. Luo, Z. S. Wang and H. H. Wen, "Superconductivity at $36 \mathrm{~K}$ in Gadolinium-Arsenide Oxides $\mathrm{GdO}_{1-\mathrm{x}} \mathrm{F}_{\mathrm{x}} \mathrm{FeAs}$," Science China Series G, Vol. 51, 2008, p. 719. doi:10.1179/143307508X333686

[13] C. Wang, L. J. Li, S. Chi, Z. W. Zhu, Z. Ren, Y. K. Li, Y. T. Wang, X. Lin, Y. K. Luo, S. Jiang, X. F. Xu, G. H. $\mathrm{Cao}$ and $\mathrm{Z}$. A. $\mathrm{Xu}$, "Thorium-Doping-Induced Superconductivity up to $56 \mathrm{~K}$ in $\mathrm{Gd}_{1-\mathrm{x}} \mathrm{Th}_{\mathrm{x}} \mathrm{FeAsO}$," Europhys. Letters, Vol. 83, No. 6, 2008, p. 67006. doi:10.1209/0295-5075/83/67006

[14] F. Han, X. Y. Zhu, G. Mu, P. Cheng and H. H. Wen, "SrFeAsF as a Parent Compound for Iron Pnictide Superconductors," Physical Review B, Vol. 78, No. 18, 2008, Id: 180503(R).

[15] H. Kabbour, L. Cario and F. Boucher, "Rational Design Of New Inorganic Compounds with the Zrsicuas Structure Type Using 2D Building Blocks," Journal of Materials Chemistry, Vol. 15, No. 34, 2005, pp. 3525-3531.

[16] X. Y. Zhu, F. Han, G. Mu, P. Cheng, B. Zeng and H. H. Wen, "Transition of Stoichiometric Sr VO FeAs to a Superconducting State at 37.2 K," Physical Review B, Vol. 79, No. 22, 2009, Id: 220512.

[17] P. S. Awana, A.Vajpayee, A. Pal, M. Mudgel, R. S. Meena and H. Kishan, "Superconductivity at $14 \mathrm{~K}$ in the Co-Doped $\mathrm{SmFe}_{0.9} \mathrm{Co}_{0.1} \mathrm{AsO}$," arXiv: 0902.3894, 2009.

[18] C. de la Cruz, W. Z. Hu, S. L. Li, Q. Huang, J. W. Lynn, M. A. Green, G. F. Chen, N. L. Wang, H. A. Mook, Q. M. Si and P. C. Dai, "Lattice Distortion and Magnetic Quantum Phase Transition in CeFeAs ${ }_{1-\mathrm{x}} \mathrm{P}_{\mathrm{x}} \mathrm{O}$," Physical Review Letters, Vol. 104, No. 1, 2009, Id: 017204.

[19] G. Wu, H. Chen, T. Wu, Y. L. Xie, Y. J. Yan, R. H. Liu, X. F. Wang, J. J. Ying and X. H. Chen, "Differentresistivityresponse to Spin-Density Wave and Superconductivity at 20 Kin Ca1-xNaxFe2As2," Journal of Physics Condensed Matter, Vol. 20, 2008, Id: 422201.

[20] A. Kreyssig, M. G. Kim, S. Nandi, D. K. Pratt, W. Tian, J. L. Zarestky, N. Ni, A. Thaler, S. L. Bud'ko, P. C. Canfield, R. J. McQueeney and A. I. Goldman, "Suppression of Antiferromagnetic Order and Orthorhombic Distortion in Superconducting $\mathrm{Ba}\left(\mathrm{Fe}_{0.961} \mathrm{Rh}_{0.039}\right)_{2} \mathrm{As}_{2}$," Physical Review $B$, Vol. 81, No. 13, 2010, Id: 134512.

[21] Z. Ren, Q. Tao, S. Jiang, C. M. Feng, C. Wang, J. H. Dai, G. H. Cao and Z. A. Xu, "Superconductivity Induced by Phosphorus Doping and Its Coexistence with Ferromagnetism in $\mathrm{EuFe}_{2}\left(\mathrm{As}_{0.7} \mathrm{P}_{0.3}\right)_{2}$," Physical Review Letters, Vol. 102, No. 13, 2008, p. 137002. doi:10.1103/PhysRevLett.102.137002
[22] J. Yang, X.-L. Shen, W. Lu, W. Yi, Z.-C. Li, Z.-A. Ren, G.-C. Che, X.-L. Dong, L.-L. Sun, F. Zhou and Z.-X. Zhao, "High- $\mathrm{T}_{\mathrm{c}}$ Superconductivity in Some Heavy Rare-Earth Iron-Asenide $\mathrm{REFeAsO}_{1-\mathrm{y}}(\mathrm{RE}=\mathrm{Ho}, \mathrm{Y}$, Dy and Tb) Compounds," Group, Vol. 11, No. 2, 2008, p. 025005 .

[23] K. Miyazawa, K. Kihou, P. M. Shirage, C.-H. Lee, H. Kito, H. Eisaki and A. Iyo, "Superconductivity above 50 $\mathrm{K}$ in LnFeAsO1y (Ln 1/4 Nd, Sm, Gd, Tb, and Dy) Synthesized by High-Pressure Technique," Journal of the Physical Society of Japan, Vol. 78, 2009, Id: 034712.

[24] P. Terzieff and K. L .Komarek, "The Paramagnetic Properties of Iron Selenides with NiAs-Type Structure," Monatshefte für Chemie, Vol. 109, No. 3, 1978, pp. 651-659. doi:10.1007/BF00912781

[25] W. Schuster, H. MiMer and K. L .Komarek, "Transition Metal-Chalcogen Systems, VII.: The Iron-Elenium Phase Diagram," Monatshefte für Chemie, Vol. 110, No. 5, 1979, pp. 1153-1170. doi:10.1007/BF00910963

[26] J. L. Yang, W. J. Ren, D. Li, W. J. Hu, B. Li and Z. D. Zhang, "Superconductivity Modulated by Internal PressureinCe $\mathrm{C}_{1-\mathrm{x}} \mathrm{Gd}_{\mathrm{x}} \mathrm{FeAsO}_{0.84} \mathrm{~F}_{0.16}$ Compounds," Superconductor Science and Technology, Vol. 23, 2010, Id: 025003 .

[27] M. Tropeano, M. R. Cimberle, C. Ferdeghini, G. Lamura, A. Martinelli, A. Palenzona, I. Pallecchi, A. Sala, I. Sheikin, F. Bernardini, M. Monni, S. Massidda and M. Putti, "Isoelectronic Ru Substitution at the Iron Site in $\mathrm{SmFe}_{1-\mathrm{x}} \mathrm{Ru}_{\mathrm{x}} \mathrm{AsO}_{0.85} \mathrm{~F}_{0.15}$ and Its Effects on Structural, Superconducting and Normal-State Properties," Physical Review B, Vol. 81, No. 18, 2010, Id: 184054.

[28] L. Wang, Y. P. Qi, Z. S. Gao, D. L. Wang, X. P. Zhang and Y. W. Ma, "The Roleof Silveraddition on the Structural and Superconducting Properties of Polycrystalline $\mathrm{Sr}_{0.6} \mathrm{~K}_{0.4} \mathrm{Fe}_{2} \mathrm{As}_{2}$," Superconductor Science and Technology, Vol. 23, 2010, Id: 025027.

[29] C. Senatore and R. Flukiger, "Upper Critical Fields Well above $100 \mathrm{~T}$ for the Superconductor SmFeAsO F with T $=46$ K," Physical Review B, Vol. 78, No. 5, 2008, Id: 054514 .

[30] L. E. Klintberg, S. K. Goh, S. Kasahara, Y. Nakai, K. Ishida, M. Sutherland, T. Shibauchi, Y. Matsuda and T. Terashima, "Chemical Pressure and Physical Pressure in $\mathrm{BaFe}_{2}\left(\mathrm{As}_{1-\mathrm{x}} \mathrm{P}_{\mathrm{x}}\right)_{2}$," Journal of the Physical Society of Japan, Vol. 79, No. 12, pp. 123706-123706-4. doi:10.1143/JPSJ.79.123706

[31] G. Mu, B. Zeng, X. Y. Zhu, F. Han, P. Cheng, B. Shen, and H. H. Wen, "Synthesis, Structural, and Transport Properties of the Hole-Doped Superconductor $\operatorname{Pr}_{1-\mathrm{x}} \mathrm{Sr}_{\mathrm{x}} \mathrm{FeAsO}$," Physical Review B, Vol. 79, No. 10, 2008.

[32] K. Kasperkiewicz, J.-W. G. Bos, A. N. Firch, K. Prassides and S. Margadonna, "Structural and Electronic Response upon Hole Doping of Rare-Earth Iron Oxyarsenides $\mathrm{Nd}_{1-\mathrm{x}} \mathrm{Sr}_{\mathrm{x}} \mathrm{FeAsO}(0<\mathrm{x} \leq 0.2)$," Chemical Communications, No. 6, 2009, pp. 707-709. doi: $10.1039 / \mathrm{b} 815830 \mathrm{~d}$

[33] Y. Mizuguhci, Y. Hara, K. Deguchi, S. Tsuda1, T. Yamaguchi, K. Takeda, H. Kotegawa, H. Tou and Y. Ta- 
kano, "Anion Height Dependence of Tc for the Fe-Based Superconductor," Superconductor Science and Technology, Vol. 23, No. 5, 2010, Id: 054013.

[34] D. Berardan, L. D. Zhao, L. Pinsard-Gaudart and N. Dragoe, "Electronic Phase Diagram of $\mathrm{Fe}_{1-\mathrm{x}} \mathrm{Rh}_{\mathrm{x}} \mathrm{AsO}$," Physical Review B, Vol. 81, 2010, Id: 094506.

[35] A. Koitzsch, D. Inosov, J. Fink, M. Knupfer, H. Eschrig, S. V. Borisenko, G. Behr, A. Köhler, J. Werner, B. Büchner, R. Follath and H. A. Dürr, "Valence-Band and Core-Level Photoemission Spectroscopy of La$\mathrm{FeAsO}_{1-\mathrm{x}} \mathrm{F}_{\mathrm{x}}$," Physical Review B, Vol. 78, No. 18, 2008, Id: 180506.

[36] X. Y. zhu, H. Yang, L. Fang, G. Mu and H. H. Wen, "Upper Criticalfield, Hall Effectand Magnetoresistance Inthe Iron-Based Layeredsuperconductor $\mathrm{LaFeAsO}_{0.9} \mathrm{~F}_{0.1-\delta}$," Superconductor Science and Technolog, Vol. 21, No. 10, 2008, ID: 105001.

[37] G. F. Chen, Z. Li, G. Li, J. zhou, D. Wu, J. Dong, W. Z. Hu, P. Zheng, Z. J. Chen, H. Q. Yuan, J. Singleton, J. L. Luo and N. L. Wang, "Superconducting Properties of the

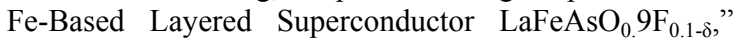
Physical Review letters, Vol. 101, No. 5, 2008, Id: 057007.

[38] A. H. Fang, F. Q. Wang and X. M. Xie, "Low-Temperature
Rapid Synthesis and Superconductivity of Fe-Based Oxypnictide Superconductors," Journal of the American Chemical Society, Vol. 132, No. 10, 2010. pp. 3260-3261. doi:10.1021/ja100055k

[39] S. Lebgue, "Electronic Structure and Properties of the Fermi Surface of the Superconductor LaOFeP," Physical Review B, Vol. 75, No. 3, 2007, Id: 035110.

[40] G. F. Chen, Z. Li, G. Li, W. Z. Hu, J. Dong, J. Zhou, X. D. Zhang, P. Zheng, N. L. Wang and J. L. Luo, "Superconductivity in Hole-Doped $\left(\mathrm{Sr}_{1-\mathrm{x}} \mathrm{K}_{\mathrm{x}}\right) \mathrm{Fe}_{2} \mathrm{As}_{2}$," Chinese Physical Letters, Vol. 25, No. 9, 2008, p. 3403. doi:10.1088/0256-307X/25/9/083

[41] J. Q. Yan, A. Kreyssig, S. Nandi, N. Ni, S. L. Bud'ko, A. Kracher, R. J. McQueeney, R. W. McCallum, T. A. Lograsso, A. I. Goldman and P. C. Canfield, "Structural Transition and Anisotropic Properties of Single-Crystalline $\mathrm{SrFe}_{2} \mathrm{As}_{2}$," Physical Review B, Vol. 78, No. 2, 2008, Id: 024516.

[42] G. F. Chen, Z. Li, J. Dong, G. Li, W. Z. Hu, X. D. Zhang, X. H. Song, P. Zheng, N. L. Wang and J. L. Luo, "Transport and Anisotropy in Single-Crystalline $\mathrm{SrFe}_{2} \mathrm{As}_{2}$ and $\mathrm{A}_{0.6} \mathrm{~K}_{0.4} \mathrm{Fe}_{2} \mathrm{As}_{2} \quad(\mathrm{~A}=\mathrm{Sr}, \mathrm{Ba})$ Superconductors," Physical Review B, Vol. 78, No. 22, 2008, Id: 224512. 